\title{
Diagnóstico prenatal de aneuploidías por amniocentesis. Experiencia de dos años de trabajo
}

\author{
Carlos Hernán Becerra Mojica MD*; Norma Cecilia Serrano Díaz, MD*; MSc **
}

\section{RESUMEN}

Se presentan los resultados de una serie de 209 mujeres embarazadas que acudieron entre 1996 y 1997 al Centro Médico Carlos Ardila Lülle de Bucaramanga, para diagnóstico citogenético prenatal. La frecuencia de pérdidas gestacionales fue del $0,46 \%$ (IC $95 \% 0.0$ a $2,55 \%$ ) y la de cultivos aptos para lectura del $95,83 \%$ (IC 95\% 92.24\% a 98.08\%), siendo mejor esta proporción en el segundo año de la serie. El $4.83 \%$ de los estudios presentaron una constitución cromosómica anormal (IC $95 \% 2.33$ a $8.70 \%$ ), en donde el hallazgo previo de una malformación por sonografía representa un riesgo 5.24 mayor de un resultado anormal. No se encontraron casos de aneuploidía en mujeres menores de 40 años, ni en aquellas que por solicitud voluntaria se les realizó el estudio.

PALABRAS CLAVES: Aneuploidía, trisomía, diagnóstico prenatal, amniocentesis, diagnóstico genético.

\section{SUMMARY}

We presented the results of a series of 209 pregnant women attended between 1996 and 1997 to the Centro Medico Carlos Ardila Lülle at Bucaramanga for cytogenetic prenatal diagnosis. The frecuency of gestacional losses was $0,46 \%$ (IC $95 \% ; 0,0$ to $2,55 \%$ ), and that of able cultivation for reading of the $95,83 \%$ (IC $95 \% ; 92,24 \%$ to $98.08 \%$ ), being this greater proportion in the second year of the series. $4,83 \%$ of the studies showed an abnormal chromosomal constitution (IC $95 \% ; 2,33 \%$ to $8,70 \%$ ), in which the previous finding of a malformation detected, by sonography represents a risk 5,24 greater than an abnormal finding as compared to the $>40$ years old women. They were not found aneuploidic cases women below 40 years old, neither in those that by request witting was them accomplished is study.

KEY WORDS: Aneuploidy, trisomy, Prenatal diagnosis, amniocentesis, Genetic diagnosis.

\section{Introducción}

La amniocentesis como herramienta utilizada para el diagnóstico genético fetal fue iniciada por Steele y Breg en 1966, demostrando por primera vez la posibilidad de realizar análisis cromosómico en las células de líquido amniótico (1); posteriormente Jacobson y Barter reportaron el primer diagnóstico prenatal de anomalía cromosómica (2). En los años siguientes se informaron diagnósticos prenatales de una amplia variedad de anomalías cromosómicas y metabólicas $(3,4)$.

Con el ultrasonido de tiempo real y las mejoras en los medios de cultivo, estas técnicas son cada vez más seguras y eficientes. En el Centro Médico "Carlos Ardila Lülle" (CMCAL) estamos realizando amniocentesis para cariotipo fetal desde finales de 1995. En este artículo se presentan los resultados obtenidos durante los primeros 2 años de trabajo en esta área del Diagnóstico Prenatal.

\footnotetext{
* Médico Perinatólogo del Complejo Centro Médico - Clínica Carlos Ardila Lülle. Profesor de la Facultad de Medicina de la Universidad Industrial de Santander, UIS.

** Médica Genetista del Complejo Centro Médico - Clínica Carlos Ardila Lülle, Laboratorio Higuera Escalante. Profesora de la Facultad de Medicina de la Universidad Autónoma de Bucaramanga, UNAB.
}

\section{Metodología}

Tipo de estudio. Se revisaron las notas clínicas de las pacientes que acudieron al CMCAL para diagnóstico prenatal de aneuploidías por medio de estudio citogenético de células procedentes de líquido amniótico, y que fueron atendidas en 1996 y 1997. De allí se captó la información correspondiente a edad de la madre, motivo del estudio, edad gestacional, número de fetos a estudiar, número de amniocentesis requeridas para obtener una muestra adecuada de líquido amniótico, complicaciones de este último procedimiento, eficacia del crecimiento celular y cariotipo resultante.

Amniocentesis. Las pacientes reciben en su primera visita información detallada acerca de las características del procedimiento, su seguridad, riesgos derivados del mismo, alcances del estudio, y tiempo en la obtención de resultados. Además se toma el dato de Rh materno y se procede a realizar la valoración sonográfica con el fin de determinar edad gestacional, condiciones fetales, cantidad de líquido amníotico y localización de la placenta. Con base en esta información se decide el momento en el cual se debe realizar el procedimiento, previa firma del consentimiento informado.

El procedimiento se realiza con el concurso del perinatólogo y genetista, de manera ambulatoria, en 
consultorio, sin la utilización de anestesia ni antibióticos profilácticos. Previa asepsia y antisepsia de la pared abdominal y con guía sonográfica permanente, se penetra la cavidad uterina por vía transabdominal con aguja desechable $22 \mathrm{G}$ y 3 1/2”. Para la penetración de la pared uterina se escoge preferiblemente una zona libre de inserción placentaria. Ubicada la aguja en cavidad amniótica se retira el mandril para aspirar $2 \mathrm{cc}$ de líquido amniótico, el cual se desecha; se realiza cambio de jeringa y se procede a la toma de la muestra para estudio, obteniéndose entre 15 a $20 \mathrm{cc}$ de líquido amniótico. La cantidad tomada depende de la edad gestacional y de la valoración previa de la cantidad de líquido $(4,5)$. Obtenida la muestra, se coloca nuevamente el mandril y se procede a retirar la aguja.

Terminado el procedimiento, se valora bienestar fetal, fetocardia y la ausencia de sangrado, explicando estos parámetros a la madre. Se incapacita a la paciente por 24 horas, tiempo en el cual debe guardar reposo, previa información sobre signos de alarma que puedan indicar complicaciones.

Estudio citogenético. La muestra de líquido amniótico se lleva de inmediato al laboratorio de genética para su procesamiento. Esta es pasada a tubos Falcon de $10 \mathrm{cc}$ para centrifugación a 1000 r.p.m. durante 10 minutos. Se retira el sobrenadante, dejando aproximadamente $1 \mathrm{cc}$ de éste, el cual es utilizado para resuspender las células; este pelet es dividido en dos frascos del cultivo Falcon de 75 cc a los cuales se les adiciona 5 cc de medio de cultivo RPMI 1640 (Sigma, St Louis, EE UU) al 20\%, con suero bovino fetal inactivado (Sigma, St Louis, EE UU), más antibióticos (una mezcla de estreptomicina, penicilina y anfotericina B). Los frascos son llevados a incubación a $37{ }^{\circ} \mathrm{C}$ y $5 \%$ de $\mathrm{CO}_{2}$

El crecimiento celular es evaluado bajo visión en el microscopio invertido, a partir del día quinto de cultivo, cuando se realiza el primer cambio de medio de cultivo; una mezcla igual a la anterior. El crecimiento de células tipo amniótico, aparece más o menos hacia el séptimo día; si no se observa este tipo de crecimiento en el día 7 u 8 de cultivo, se debe repetir la muestra. Observada la proliferación celulaŕ se procede a realizar un nuevo cambio de medio, y se continúa la evaluación hasta observar la formación de un número adecuado de colonias, las cuales deben formar una monocapa. Durante este período de proliferación celular rápida, el medio debe ser cambiado cada tercer día, dependiendo del crecimiento celular.

Cuando el número de colonias es el adecuado, se procede a la obtención de la cosecha (6). Las láminas son procesadas para banda $G$ (7), y se realiza la evaluación final de las metafases y fotografía de las mismas, bajo visión en un fotomicroscopio de luz directa.

Obtenido en resultado citogenético, la madre es vista en control para la entrega y explicación de éste, y valoración sonográfica de bienestar fetal y cantidad de líquido amniótico.

Posterior al parto, la madre es citada a control con su hijo, para evaluación clínica del recién nacido.

Análisis estadístico. La información recolectada se transcribió en una base de datos en Epi Info 6.04b para análisis descriptivo univariado de las madres, de las amniocentesis y de los cultivos celulares. En los casos de las proporciones, se calculó su intervalo de confianza al 95\% (IC 95\%) y en el de las variables numéricas, la media y desviación estándar $(X+/-D E)$.

Posteriormente se hizo análisis bivariado entre el éxito en el crecimiento del cultivo celular, frente a los demás posibles determinantes, así como entre éstos y un resultado de cariotipo negativo. En el caso de los embarazos múltiples, cada producto se analizó como si fuera un producto independiente. Para las diferencias entre crecimiento exitoso y fallido, así como entre cariotipo normal y anormal, se calculó el riesgo relativo existente, junto con sus intervalos de confianza al $95 \%$, teniendo en cuenta para aceptación de significancia estadística, una probabilidad límite del $5 \%$.

\section{Resultados}

Se atendieron 209 madres, con edades entre 17 y 44 años (34.58 \pm 0.42 años). El $57.42 \%$, entre 35 y 44 años (Figura 1), corresponde a 8.7 mujeres al mes: una cada 3,49 días. Tres de las 209 madres tenían embarazo gemelar (1.44\% IC 95\% 0.30 a $4.14 \%$ ), siendo así 212 productos estudiados. Ocho de las concepciones eran producto del programa de fertilización in vitro, IVF (3.82\%, IC $95 \% 1.67$ a $7.49 \%)$. En más de la mitad de las mujeres, el motivo de la amniocentesis y del diagnóstico citogenético, fue la edad avanzada (Figura 2).

Se realizaron en total 216 amniocentesis, siendo la edad gestacional a la que se practicó la prueba, de 14 a 33 semanas ( $16.99 \pm 0.18$ semanas), estando el $51.39 \%$ entre semanas 14 y 16, como se ve en la figura 3. En sólo un caso, fue necesario puncionar 2 veces para obtener la muestra. Como complicación se presentó la pérdida de una gestación $(0.46 \%$ para ambas situaciones; IC $95 \%$ 0.0 a $2.55 \%$ )

Inicialmente se realizaron 212 amniocentesis, una por cada feto, de las cuales en 203 se logró cultivo adecuado para estudio citogenético (proporción de crecimiento al primer intento del $95.75 \%$; IC 95\% 92.09 a $98.04 \%$ ). En el primer año de la serie, se presentaron más fracasos en el crecimiento celular (6/97, 6.19\%; IC 95\% 2.30 a $12.98 \%$ ) que en el segundo (3/122, $2.46 \%$; IC $95 \%$ 0.51 a $7.02 \%$ ). Cuatro de las nueve madres en las que no se obtuvo crecimiento en el primer intento, se sometieron a una segunda amniocentesis, creciendo las células en todos los casos, con lo cual, en total, se obtuvo crecimiento celular del $95.83 \%$ (IC $95 \% 92.24 \%$ a $98.08 \%$ ) de todos los líquidos sembrados, siendo finalmente 207 cultivos adecuados para lectura y análisis del cariotipo.

El cariotipo fue normal en 206 productos $(95.17$; IC $95 \% 91.30$ a $97.67 \%) ; 92$ (44.44\%; IC 95\% 37.56 a $51.49 \%$ ) tenía una constitución cromosómica $46, \mathrm{XX}$ y 155 (55.56\%; IC 95\% 48.51 a $62.44 \%$ ) 46,XY. Los 10 estudios anormales correspondieron todos a trisomías, 9 de ellos por trisomía libre y universal y uno producto de translocación: 5 trisomías 21, 4 trisomías 18 y una trisomía 13 por $\mathrm{t}(13 \mathrm{p}, 14 \mathrm{p})$.

Ninguna de las variables disponibles, se asoció de manera significativa con éxito o fracaso del crecimiento 
Figura 1

EDAD DE LA PACIENTE

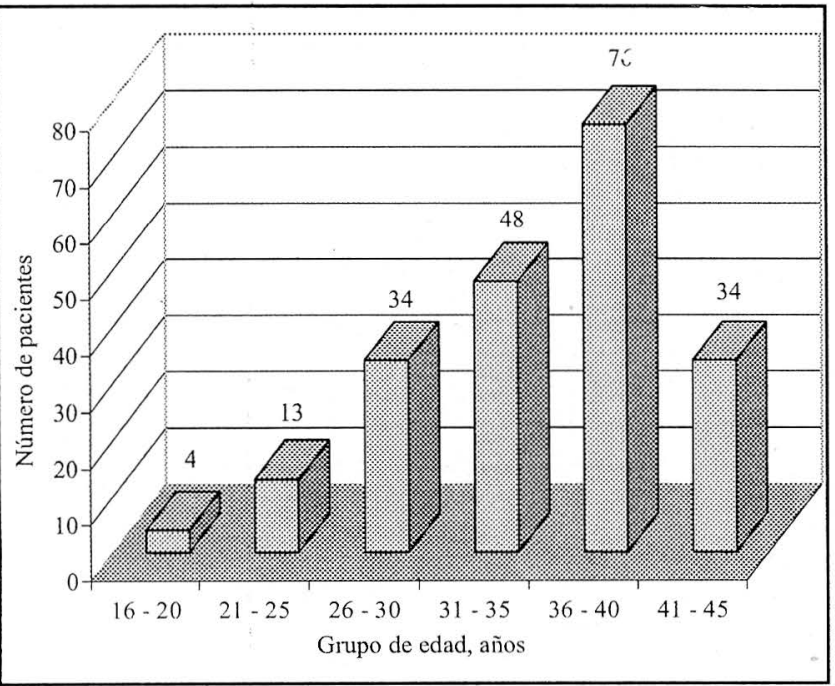

Figura 2

MOTIVO DEL ESTUDIO

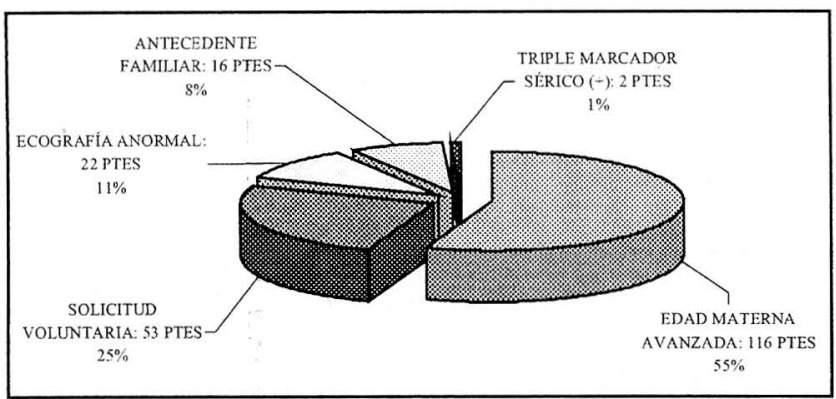

Tabla 1.

ASOCIACION ENTRE CULTIVO EXITOSO Y MOTIVO DEL ESTUDIO

\begin{tabular}{|lcccc|}
\hline Factor estudiado & Cultivo exitoso & Cultivo negativo & RR (IC 95\%) & $p$ \\
\hline Cultivos & 207 & 9 & - & - \\
Edad materna (años) & $34.53 \pm 6.10$ & $36.78 \pm 4.87$ & - & 0.276 \\
Edad gestacional (semanas) & $17.01 \pm 2.72$ & $16.56 \pm 1.59$ & - & 0.620 \\
Gestación por FIV & $8(3.86 \%)$ & $0(0 \%)$ & $1.05(1.02$ a 1.08$)$ & 0.549 \\
Embarazo gemelar & $6(2.90 \%)$ & $0(0 \%)$ & $1.04(1.02$ a 1.08$)$ & 0.605 \\
Motivo de consulta* & & & & \\
Edad materna avanzada & $117(95.12 \%)$ & $6(4.88 \%)$ & - & 0.900 \\
Voluntaria & $51(96.23 \%)$ & $2(3.77 \%)$ & & \\
Eco anormal & $21(95.45 \%)$ & $1(4.55 \%)$ & & \\
Antecedente & $16(100 \%)$ & $0(0 \%)$ & & \\
Triple marcador $(+)$ & $2(100 \%)$ & $0(0 \%)$ & \\
\hline \multicolumn{4}{c}{ * Proporción calculada sobre la base del total de madres que acudieron por el motivo de consulta enunciado } \\
\end{tabular}

Tabla 2.

ASOCIACION ENTRE CARIOTIPO NORMAL Y MOTIVO DEL ESTUDIO

\begin{tabular}{|c|c|c|c|c|}
\hline \multirow[b]{2}{*}{ Factor estudiado } & \multicolumn{2}{|c|}{ Cariotipo } & \multirow[b]{2}{*}{ RR (IC 95\%) } & \multirow[b]{2}{*}{$p$} \\
\hline & Normal & Anormal & & \\
\hline Cultivos & 197 & 10 & - & - \\
\hline Edad materna (años) & $34.50 \pm 6.00$ & $34.10 \pm 8.19$ & - & 0.761 \\
\hline Edad gestacional (semanas) & $16.95 \pm 2.66$ & $18.20 \pm 3.65$ & - & 0.157 \\
\hline Gestación por FIV & $8(4.57 \%)$ & $0(0 \%)$ & $1.05(1.02$ a 1.09$)$ & 0.668 \\
\hline Embarazo gemelar & $6(3.05 \%)$ & $0(0 \%)$ & $1.06(1.02$ a 1.09$)$ & 0.729 \\
\hline Cultivo segunda muestra & $4(2.03 \%)$ & $0(0 \%)$ & $1.05(1.02$ a 1.09$)$ & 0.811 \\
\hline Cariotipo XX & $89(45.18 \%)$ & $3(30.0 \%)$ & $1.03(0.97$ a 1.09$)$ & 0.273 \\
\hline \multicolumn{5}{|l|}{ Motivo del estudio* } \\
\hline Edad materna avanzada & $105(95.45 \%)$ & $5(4.55 \%)$ & - & 0.000 \\
\hline Voluntaria & $51(100 \%)$ & $0(\quad 0 \%)$ & & \\
\hline Eco anormal & $16(76.19 \%)$ & $6(23.81 \%)$ & & \\
\hline Antecedente & $16(100 \%)$ & $0(0 \%)$ & & \\
\hline Triple marcador $(+)$ & $2(100 \%)$ & $0(\quad 0 \%)$ & & \\
\hline
\end{tabular}

* Proporción calculada sobre la base del total de madres que acudieron por el motivo de consulta enunciado

Figura 3

EDAD GEST ACIONAL

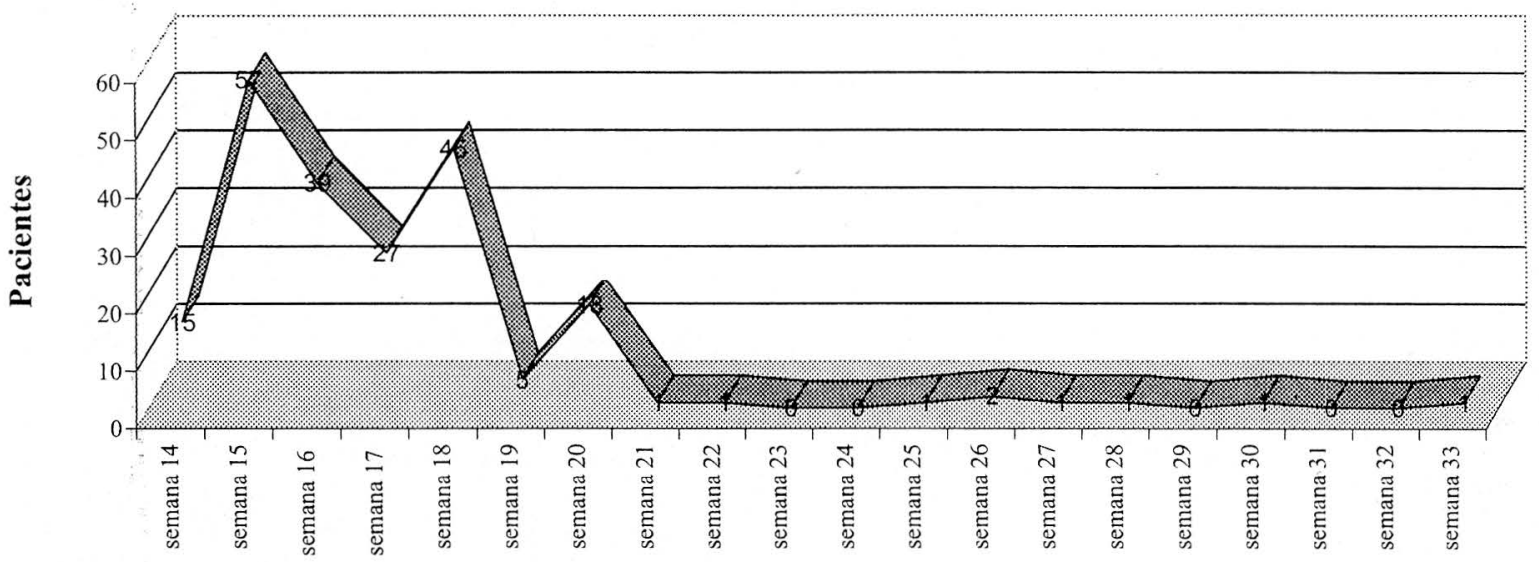

Edad Gestacional, semanas 
celular, como se aprecia en la Tabla 1. Tan sólo el hecho de haberse realizado el estudio citogenético por hallazgo sonográfico anormal durante el control prenatal, se asoció con cariotipo anormal (Tabla 2, p < 0.00068), significando un riesgo de 5.24 (IC 95\% 1.66 a 16.52) veces más de resultar anormal frente a los casos en los que el estudio se hizo por edad materna avanzada $(p<0.0024)$.

Ahora bien, cuando se contempla la relación que existe entre edad y cariotipo anormal, se encuentra que entre las 161 mujeres que consultaron voluntariamente o por edad avanzada todos los resultados citogenéticos anormales, se dieron entre las madres con 40 o más años, ya que en ninguna de las 108 mujeres menores de esa edad, se encontró un resultado adverso $(0 \%$; IC $95 \% 0.0$ a $3.36 \%$ ), mientras que 5 de las 53 mujeres con 40 o más años, si lo tuvieron $(9.43 \%$; IC $95 \% 3.13$ a $20.66 \%$; p < $0.0058)$.

\section{Discusión}

La amniocentesis es un procedimiento ampliamente usado en el diagnóstico prenatal de las alteraciones cromosómicas fetales. Luego de revisar nuestra experiencia, encontramos semejanzas en cuanto a la edad de las madres motivo de estudio, comparado con otros informes, similitud dada por tratarse del principal motivo de consulta, la edad mayor a 35 años, parámetro establecido como un riesgo mayor de aneuploidías $(8,9)$.

El momento del examen, se correlaciona con el motivo de consulta, entre 15 a 16 semanas, para las pacientes que consultan por edad materna avanzada, y a mayor edad gestacional, cuando es orientado el estudio por hallazgos sonográficos anormales.

El porcentaje de complicaciones relacionadas con el procedimiento, es bajo $(0.46 \%)$, comparado con lo informado en otros,centros $(8,10,11)$. En este estudio se trató de una paciente tosedora crónica quien presentó ruptura de las membranas, con pérdida de la gestación. La eficacia del procedimiento está demostrada por el hecho de que en todos los casos, se obtuvo la muestra deseada y solo en un caso, al inicio de la serie, fue necesario realizar dos punciones, mostrando cómo la experiencia del operador, es un factor determinante.

De las 216 amniocentesis realizadas, se logró crecimiento celular apto para lectura y análisis citogenético en el $93.81 \%$, durante el primer año de trabajo, y de $97.54 \%$ durante el segundo, porcentajes que están por ahora por debajo de los reportados en los grandes centros de diagnóstico citogenético prenatal $(4,9,12)$, pero que muestran un mejoramiento en la medida en que se realizan más cultivos y las condiciones de laboratorio se logran estandarizar.

El porcentaje de aneuploidías $(4.86 \%)$, coincide con lo informado en otros centros $(9,13,14)$. Las alteraciones cromosómicas halladas en el grupo de edad materna avanzada, se presentaron todas en pacientes mayores a 40 años, porque el riesgo es mayor a medida que avanza la edad de la madre gestante $(8,9)$.

El mayor porcentaje de aneuploidías fue hallado en aquellas pacientes donde se decidió hacer estudio citogenético por encontrar marcadores sonográficos positivos, lo cual indica que la combinación de estas dos herramientas, sonografía y estudio citogenético fetal, hacen que aumente la eficacia del diagnóstico prenatal.

No podemos informar acerca de la precisión de los estudios citogenéticos, dado que más de la mitad de las pacientes no regresaron para la evaluación clínica del recién nacido. En los que se realizó dicha evaluación, los hallazgos clínicos fueron de total concordancia con los citogenéticos.

La presentación de los resultados del trabajo realizado durante dos años, nos permite concluir que la amniocentesis es un procedimiento seguro para la paciente y el feto y que las muestras de líquido amniótico obtenidas, tienen un crecimiento celular eficaz, lo cual hace que el análisis citogenético de éstas sea confiable. Finalmente tenemos un soporte científico que respalda el trabajo y permite dar una información veraz a la paciente, basados en la evaluación de la experiencia de trabajo.

\section{BIBLIOGRAFIA}

1. Steele, M. W., Breg, W. R. Jr. Chromosome analysis of human amniotic fuid cells. Lancet 1966; 1: 388.

2. Jacobson, C.C., Barter, R. H. Intrauterine diagnosis and management of genetic difects. Am J Obstet Gynecol 1967; 99: 795.

3. Nadler, H. L. Antenatal detection of hereditary disorders. Pediatrics 1968; 42: 912.

4. Aubrey Milunsky. MB. B. Ch. Genetic disorders and the Fetus. The Johns Hopkins University Press 1992: 33-101.

5. Grether, P., Zavalero, M., De la Luna, E. Diagnóstico Prenatal en 350 aminocenteis. Ginec, Obst. Mex 1991; 59: 317-322.

6. Wolf, U. Culture and preparation of cell from amnotic fluid. In: Scwarzacher HG, Wolf U, Passarge E. (Eds). Methods in human cytogenetics. Springer Veriag, New York 1974.

7. Seabright, M. A rapid banding technique for human chromosomes. Lancet1971; 2: 971.
8. Golbus, M. S., Loughman, W. D., Epstein, Ch. J. Prenatal genetc diagnosis in 300 Amniocenteis. N. Engl. J. Med 1979; 300: 157-163.

9. Working, P. Amniocentesis an assessment of hazards of amniocentesis. Br. J. Obstet, Gynec 1985; 1: 878 Suppl 2:1.

10. Crandall, B. F., Howard, J., Thomas, B. L. Felow - up of 2000 second - trimester. Amniocenteis. Obstet Gynecol 1980; 56: 625.

11. Andre Bové. Fetal Medicine. Prenatal diagnosis and Managemente. Oxfor University Press 1995: 26-47.

12. Milunsky, A., y Atkins, L. The frequency of chromosome abnormalities diagnosed prenatally. En: Population cytogenetics studies in humans, Hook, E. B. Y Porter, I. H. (Eds.). Academic Press, New York 1977: 11-25.

13. Salamanca, F., Buentello, L., y Canún, S. Estudio citogenético en 5.125 recién nacidos consecutivos en la ciudad de México. En: Estudios de antropología biológica. U.N.A.M., México, D.F., 1982: 125-140. 